\title{
Application of Artificial Neural Networks in Weather Forecasting: A Comprehensive Literature Review
}

\author{
Gyanesh Shrivastava \\ Dr. C.V. Raman University, \\ Bilaspur, Chhattisgarh, India
}

\author{
Sanjeev Karmakar \\ Bhilai Institute of Technology (BIT) \\ Bhilai House, Durg, 491001, \\ Chhattisgarh, India \\ Pulak Guhathakurta \\ India Meteorological Department (IMD) \\ Sivaji Nagar, Pune, India
}

\author{
Manoj Kumar Kowar \\ Bhilai Institute of Technology (BIT) \\ Bhilai House, Durg, 491001, \\ Chhattisgarh, India
}

\begin{abstract}
To recognize application of Artificial Neural Networks (ANNs) in weather forecasting, especially in rainfall forecasting a comprehensive literature review from 1923 to 2012 is done and presented in this paper. And it is found that architectures of ANN such as BPN, RBFN is best established to be forecast chaotic behavior and have efficient enough to forecast monsoon rainfall as well as other weather parameter prediction phenomenon over the smaller geographical region.
\end{abstract}

\section{Keywords}

ANN, BPN, Back-propagation, RBF, Rainfall, Forecasting.

\section{INTRODUCTION}

Weather forecasting (especially rainfall) is one of the most important and challenging operational tasks carried out by meteorological services all over the world. It is furthermore a complicated procedure that includes multiple specialized fields of expertise. Researchers in this field have separated weather forecasting methodologies into two main branches in terms of numerical modeling and scientific processing of meteorological data. The most widespread techniques used for rainfall forecasting are the numerical and statistical methods. Even though researches in these fields are being conducted for a long time, successes of these models are rarely visible. There is limited success in forecasting the weather parameters using the numerical model. The accuracy of the models is dependent upon the initial conditions that are inherently incomplete. These systems are not able to produce satisfactory results in local and short-term cases. The performances, however, are poor for long range prediction of monsoon rainfall even for the larger spatial scale and particularly, for the Indian region. As an alternative, statistical methods in which rainfall time series are treated as stochastic are widely used for long-range predication of rainfall. IMD has been using statistical models for predicting monsoon rainfall. Statistical models were successful in those years of normal monsoon rainfall and failed remarkably during the extreme monsoon years like 2002 and 2004. However, it is very difficult to get the same or better skill in predicting district level monsoon rainfall as that of all-India level monsoon rainfall using these statistical models. Two main drawbacks of these statistical models are:

1. Statistical models are not useful to study the highly nonlinear relationships between rainfall and its predictors, even if one considers models like power regression.

2. There is no ultimate end in finding the best predictors. It will never be possible to get different sets of regional and global predictors to explain the variability of the two neighbouring regions having distinguished rainfall features.

Karmakar et al., 2008,2009, has found that, the variability of monsoon rainfall over the Indian district level or very small scale geographical region is due to external forcing i.e., global weather parameter and also the internal variability within the time series itself. If external forcing is assumed to be same then also there remains variability within the time series that can only be explained if we are able to predict the internal dynamical behavior of the weather data time series. The pattern recognition and prediction in a deterministic approach through ANN technique based on back-propagation algorithm is found to be the most efficient way by which internal dynamics of rainfall time series data has been successfully identified. Moreover, pattern recognition and prediction have a longer lead-time as they can be made a year in advance. No other model (except the neural network model) so far has been able to forecast district level long-range weather parameter so accurately.

Artificial Neural Network came into existence in 1986 which is able to get rid of the above two drawbacks and drawing considerable attention of research workers, as it can handle the complex non-linearity problems better than the conventional existing statistical techniques.

The objective of this study is to expand and evaluate the ANN. This objective is considered via comprehensive review of literature (1923-2012). It is found that, detail of discussion concerning the architecture of ANN for the same is rarely visible in the literature; however various applications of ANN are available. The paper has been constructed with the sections. Section II discussed a comprehensive review of world-wide contribution from 1923 to 2012 . Methodology to forecast those are unquestionably accepted and no scientific controversy to forecast rainfall has been discussed in section III. Result of the review has been discussed in section IV and finally a conclusion is discussed in the section $\mathrm{V}$.

\section{LITERATURE REVIEW}

After initial work of Walker [1,2] several attempts by Gowariker et al., [3,4], Thapliyal et al., [5,6] have been made for developing better models for long-range forecasts of summer monsoon rainfall in India. Performance of the Gowariker et al., [4]; Rajeevan et al., [7,8], Thapliyal et al., [9] regression models based on different sets of predictors have found to be satisfactory and reasonably accurate during last eleven years. These models are being extensively used by IMD for long-range forecasts of summer monsoon (June-September) rainfall over India as a whole. Recently, IMD has been trying to forecast for Indian sub regions and issuing long-range forecasts for three broad 
homogeneous regions of India, viz., Northwest India, Northeast India and the Peninsula through the updated three individual power regression models based on different sets of predictors [10]. However, Guhathakurta [11] found these statistical models to be successful in those years of normal monsoon rainfall and failed remarkably during the extreme monsoon years like 2002 and 2004. Also Rajeevan et al., Thapliyal et al., [5, 6, 8-10] have found that the statistical models have many inherent limitations. Guhathakurta et al., [12] have observed that the correlations between monsoon rainfall and the predictors can never be perfect and there is no ultimate end in finding the best predictors. Parthasarathy et al., Hastenrath et al., $[13,14]$ have found they may suffer epochal changes and there may be crosscorrelations between the parameters. Rajeevan et al., Guhathakurta, Krishnamurthy et al., Sahai et al., [9,15-18] have found that attempts to forecast monsoon rainfall as well as climate parameters through statistical technique over smaller areas like a district, or monsoon periods such as a July, monsoon (June-September), have become unsuccessful as correlations fall drastically. Guhathakurta $[19,20]$ has observed that the weather prediction over high-resolution geographical regions is very complicated. However, since 1986, the ANN technique has been drawing considerable attention of research workers, as it can handle the complex non-linearity problems better than the conventional existing statistical techniques.

In the case study of The chaotic time series of Indian monsoon rainfall, Basu and Andharia,1992,have found that the resulting forecast formula uses only the rainfall of past seven years as predictors, making a forecast eight months in advance[21].

After development of recurrent Sigma- Pi neural network rainfall forecasting system, Chow and Cho, 1997, have concluded that the neural network based now casting system is capable of providing a reliable rainfall now casting in Hong Kong [22].

Lee et al., 1998, have found that RBF networks produced good prediction while the linear models poor prediction [23]. Hsieh, W.H. and B. Tang, 1998, applied various ANN models for prediction and analysis in meteorology data as well as oceanography data and have found ANN technique is extremely useful [24]. In another research Dawson and Wilby have found that, rainfall runoff modeling, the ability of the ANN to cope with missing data and to "learn" from the event currently being forecast in real time makes it an appealing alternative to conventional lumped or semi distributed flood forecasting models [25]. Guhathakurta et al., have found that performance of the hybrid model (model III), has been the best among all three models developed. RMSE of this hybrid model is $4.93 \%$. As this hybrid model is showing good results it is now used by the IMD for experimental long range forecast of summer monsoon rainfall over India as a whole [26].

Ricardo et al., 1999, have used this technology for simulation of daily temperature for climate change over Portugal [27]. Wherein, performances of linear models and non-linear ANN are compared using a set of rigorous validation techniques. Finally, the non-linear ANN model is initialized with general circulation model output to construct scenarios of daily temperature at the present day (1970-79) and for a future decade (2090-99). Charles Jones and Pete Peterson, 1999, [28] have completed a research at the University of California, Santa Barbara, California, for air surface temperature prediction over the city. Guhathakurta, 1999, [29,30] has implemented this technique for short-term prediction of surface ozone at Pune city. In this work multiple regression data analysis using ANN technique has been used. It has been observed that, the parallel model can be developed for all the major cities with different sets of related data but the network architecture will be different.

After comparative study of short term rainfall prediction models for real time flood forecasting, E. Toth et al., have found that the time series analysis technique based on ANN provides significant improvement in the flood forecasting accuracy in comparison to the use of simple rainfall prediction approaches [31]

In 2001 Luk et al., have developed and compared three types of ANNs suitable for rainfall prediction i.e. multilayer feed forward neural network, Elman partial recurrent neural network and time delay neural network [32]. Michaelides et al., have found that ANN is a suitable tool for the study of the medium and long term climatic variability. The ANN models trained were capable of detecting even minor characteristics and differentiating between various classes [33]. After a study of RBFNN, Chang et al., 2001, have found that RBFNN is a suitable technique for a rainfall runoff model for three hours ahead floods forecasting [34].

Brath et al., 2002, have presented time series analysis technique for improving the real time flood forecast by a deterministic lumped rainfall runoff model and they have concluded that apart from ANNs with adaptive training, all the time series analysis techniques considered allow significant improvements if flood forecasting accuracy compared with the use of empirical rainfall predictors [35]. Using ANN for daily rainfall runoff modeling, Rajurkar et al., have found that coupling of ANN with a multiple-input single-output model predicted the daily runoff values with high accuracy both in the training and validation periods [36]. For a rainfall runoff relationship Harun and Irwan, 2002, have concluded that the performance of neural network model is better than HEC-HMS and MLR models for modeling the rainfall runoff relationship [37]. Iseri et al., 2002, have developed medium term forecasting of August rainfall in Fukuoka city. In order to identify the sufficient predictors, the partial mutual information was used for the candidate predictors, which are Sea Surface Temperature anomalies (SSTa) in the Pacific Ocean and three climate indices. When data with lead times between one and twelve months were used to forecast August rainfall, it was found that a model with the North Pacific index and selected SSTa as inputs performed reasonably well [38].

Silva and Snell et al., 2003, have applied this approach both in terms of predictive accuracy and model encompassing. This technology is currently being widely applied to climate prediction because of its ability to explain the complex behavior through time series as well as regression data analysis $[39,40]$. Richard, 2003, has completed simulation of European climate, through this technique. In that study, Neural Network was used for linear regression analysis [41].

In 2004 Maqsood et al., have found that HFM is relatively less accurate and RBFN is relatively more reliable for the weather forecasting problems and in comparison the ensembles of neural networks produced the most accurate forecast [42]. After applying soft computing techniques Pasero and Moniaci, 2004, have found that the system is able to forecast the evolution of the parameters in next three hours giving previous indications about the possibility of rain, ice and fog [43]. Lekkas et al., 2004 have used a multilayer back propagation network and found that BPN will not always find the correct weight and biases fort the optimum solution, whereas their results supported the hypothesis that ANNs can produce qualitative forecast. A 7 hour ahead forecast in particular proves to be of fairly high precision, 
especially when an error prediction technique is introduced to the ANN model [44]. Chang Shu and Donald H. Burn, 2004, have found that artificial neural network ensembles generate improved flood estimates and are less sensitive to the choice of initial parameters when compared with a single artificial neural network [45]. In 2004 Nayak et al., have presented the application of an adaptive neuro-fuzzy inference system (ANFIS) to hydrologic time series modeling, and it was observed that the ANFIS model preserves the potential of the ANN approach fully, and eases the model building process [46]. M.Asce et al., 2004, have applied two ANN-hydrologic forecasting models and they found encouraging results indicating that ANN-hydrologic forecasting models can be considered an alternate and practical tool for stream-flow forecast [47]. In 2004 R.E. Abdel-Aal, studied alternative abductive networks approach, and concluded that the performance is significantly superior to naive forecasts based on persistence and climatology [48]. In coastal areas it is an enormous prediction of tidal level, TsongLin Lee, 2004, has predicted long-term tidal level using back propagation neural network, as compare to conventional harmonic method, he concluded that back-propagation neural network mode also efficiently predicts the long-term tidal levels [49].

To estimate the maximum surface temperature and relative humidity a Feed forward multi-layered artificial neural network model is designed by Chaudhuri, and Chattopadhyay, in 2005, and stated that one hidden-layer neural network is an efficient forecasting tool by which an estimation of maximum surface temperature and maximum relative humidity can be obtained [50]. A further contribution of Gwo-Fong Lin* and Lu-Hsien Chen, 2005, in neural network is that, two hidden layers is developed to forecast typhoon rainfall, and it has been observed that the forecasting model can produce reasonable forecasts [51]. Using an innovation in the researches Jon Vandegriff et al., 2005, have studied Forecasting space weather with ANN and they found that an ANN can be trained to predict the shock arrival with better accuracy than existing methods [52]. Ozgur KISI, 2005, has selected three simple neural network (NN) architectures, i.e. ANN, Auto-Regressive Models and sum of square errors, for comparison of forecasting probabilities and he found that $\mathrm{NNs}$ were able to produce better results than $\mathrm{AR}$ models when given the same data inputs [53]. Exploring the new concept, soft computing models based on Radial Basis Function Network for 24-h weather forecasting, Maqsood et al., have concluded that the RBFN produces the most accurate forecasts compared to the MLP, ERNN and HFM [54].

In 2006, Somvanshi et al., have proved that ANN model can be used as an appropriate forecasting tool to predict the rainfall, which out performs the ARIMA (Autoregressive Integrated Moving Average) model [55]. As in the previous researches we have observed that most of the researchers have been used Artificial Neural Network for various annual predictions like rainfall, tide, temperature etc, but in this study Niravesh Srikalra and Chularat Tanprasert have used Artificial Neural Network for daily rainfall prediction in Chao Phraya River with Online Data Collection, and they found that it is possible to predict rainfall on daily basis with acceptably accuracy using Artificial Neural Network [56]. A.D. Kumarasiri and D.U.J. Sonnadara, 2006, have applied an innovative technique for rainfall forecasting using Artificial Neural Networks based on feedforward back-propagation architecture. Three Neural Network models were developed; a one-day-ahead model for predicting the rainfall occurrence of the next day, which was able to make predictions with a $74.25 \%$ accuracy, and two long term forecasting models for monthly and yearly rainfall depth predictions with $58.33 \%$ and $76.67 \%$ accuracies within a $5 \%$ uncertainty level [57]. D. Nagesh et al., 2006, have used Artificial Intelligence techniques for forecasting regional rainfall and they found that this technique shows reasonably good accuracy for monthly and seasonal rainfall forecasting [58]. Guhathakurta, 2006, developed a model for rainfall forecast for the Kerala sub-division based on the area weighted value of all district forecast. The performance was found satisfactory than the statistical technique [59].

As per the utmost necessities of the hydrologists around the globe, Bustami et al., 2007, have studied ANN for precipitation and water level prediction; they found that ANN is an effective tool in forecasting both missing precipitation and water level data [60]. Paras et al., 2007, have introduced a pioneering Feature Based Neural Network Model for Weather Forecasting and the results were very encouraging and it is found that the feature based forecasting model can make predictions with high degree of accuracy [61]. Mohsen Hayati, and Zahra Mohebi, 2007, have used ANN in a new experiment of short term temperature forecasting(STTF) and he found that MLP network has the minimum forecasting error and can be considered as a good method to model the STTF systems [62]. Using time series of draught indices with artificial neural network Morid et al., 2007, have tested number of different ANN models for both Effective Drought Index (EDI) and the Standard Precipitation Index (SPI) with the lead times of 1 to 12 months [63]. As load forecasting is an important prediction aspect for industrial sectors all over the world, Mohsen Hayati, and Yazdan Shirvany, 2007, have put in an approach for short term load forecasting (STLF) using Artificial Neural Network, and they concluded that MLP network has the minimum forecasting error and can be considered as a good method to model the STLF systems [64]. To apply reliable and robust procedures for monthly reconstruction of precipitation time series, Lucio et al., 2007, have found that ANN can be applied to explore the spatiotemporal dependence of meteorological attributes [65]. In another experiment Hartmann et al., 2007, have found that the neural network algorithms are capable of explaining most of the rainfall variability even it can predict the summer rainfall also [66].

Aliev et al., 2008, have proposed, fuzzy recurrent neural network (FRNN) based time series forecasting method for solving forecasting problems, in an experiment and they found that The performance of the proposed method for forecasting fuzzy time series shows its high efficiency and effectiveness for a wide domain of application areas ranging from weather forecasting to planning in economics and business [67]. Chattopadhyay and Chattopadhyay, 2008, have worked out to find out best hidden layer size for three layered neural net in predicting monsoon rainfall in India, and they have found that eleven-hidden-nodes three-layered neural network has more efficacy than asymptotic regression in the present forecasting task [68]. Hung et al., 2008, have developed a new ANN model for forecasting rainfall from 1 to $6 \mathrm{~h}$ ahead at 75 rain gauge stations in the study area as forecast point from the data of 3 consecutive years (1997-1999), and they observed that the developed ANN model can be used for real-time rainfall forecasting and flood management [69]. In an comparative study between Artificial Intelligence and Artificial Neural Network for rainfall runoff modeling, Aytek et al.,2008, have found that genetic programming (GP) formulation performs quite well compared to results obtained by ANNs and is quite practical for use. It is concluded from the results that GEP can be proposed as an alternative to ANN models [70]. Chattopadhyay et al.,2008, have studied the complexities in the relationship between rainfall and sea surface temperature (SST) anomalies during the winter monsoon using scatter plot matrices and autocorrelation functions, and they found that the statistical 
assessment revealed the potential of artificial neural network over exponential regression [71]. However Mar, and Naing, 2008, have tested more over 100 cases by changing the number of input and hidden nodes from 1 to 10 nodes, respectively, and only one output node in an optimum artificial neural network architecture and they concluded that 3 inputs-10 hiddens- 1 output architecture model gives the best prediction result for monthly precipitation prediction [72].

Karmakar et.al., 2008, et al., 2008, have developed the ANN models for Long-Range Meteorological Parameters Pattern Recognition over the Smaller Scale Geographical Region and the performances of these models in pattern recognition and prediction have been found to be extremely good [74].

Hocaoglu et al., 2009, have developed adaptive neuro-fuzzy inference system for missing wind data forecasting [75]. In a Case Study on Jarahi Watershed, Karim Solaimani, 2009, has studied Rainfall-runoff Prediction Based on Artificial Neural Network and he found that Artificial Neural Network method is more appropriate and efficient to predict the river runoff than classical regression model [76]. KOŠCAK et al., 2009, have compared common meteorological forecasting method with ANN and he found the performance of ANN with high accuracy [77]. Karamouz et al., 2009, have experimented to perform long lead rainfall forecasting Using Statistical Downscaling and Artificial Neural Network Modeling; finally they found that the SDSM outperforms the ANN model [78]. In a comparative study between ASTAR and ARIMA methods for rainfall forecasting in Indonesia Otok, and Suhartono, 2009, have concluded that the best model is ASTAR model both in sample and out-sample data [79].

It can be well-known that neural network can applied for most of the prediction aspects, Nekoukar et al., 2010, have used radial basis function neural network for financial time-series forecasting, and the result of their experiment shows the feasibility and effectiveness [80]. Weerasinghe et al., 2010, have tested the performance of neural network, in an experiment, for forecasting daily precipitation using multiple sources, A cluster of ten neighboring weather stations having 30 years of daily precipitation data $(1970-1999)$ was used in training and testing the models. Twenty years of daily precipitation data were used to train the networks while ten years of daily precipitation data were used to test the effectiveness of the models. They found that the models were able to predict the occurrence of daily precipitation with an accuracy of $79 \pm 3 \%$ and Fuzzy classification produced a higher accuracy in predicting 'trace' precipitation than other categories [81]. Luenam et al, , 2010 have presented a Neuro-Fuzzy approach for daily rainfall prediction, and their experimental results show that overall classification accuracy of the neuro-fuzzy classifier is satisfactory [82]. Wu et al., 2010 have attempted to seek a relatively optimal data-driven model for Rainfall time series forecasting using Modular Artificial Neural Networks, they found that the normal mode indicate, MANN performs the best among all four models, but the advantage of MANN over ANN is not significant in monthly rainfall series forecasting [83]. To predict the intensity of rainfall using artificial neural network Nastos et al., 2010 have developed prognostic models and they have proved that the results of the developed and applied ANN models showed a fairly reliable forecast of the rain intensity for the next four months [84]. Patil and Ghatol, 2010, have used various ANN topologies such as radial basis functions and multilayer perceptron with Levenberg Marquardt and momentum learning rules for predicting rainfall using local parameters and they found the topologies fit for the same task
[85]. Tiron, and Gosav, 2010, have estimated rainfall from BARNOVA WSR-98 D Radar using Artificial Neural Network and the efficiency of ANN in the estimation of the rain rate on the ground in comparison with that supplied by the weather radar is evaluated [86]. Goyal and Ojha, 2010, have focused their working on a concept of using dimensionless variables as input and output to ANN, finally they have concluded that ANN model using dimensionless variables were able to provide a better representation of rainfall-runoff process in com-parison with the ANN models using process variables investigated in this study [87]. On the basis of humidity, dew point and pressure in India, Enireddy et al., 2010, have used the back propagation neural network model for predicting the rainfall. In the training they have obtained $99.79 \%$ of accuracy and $94.28 \%$ in testing. From these results they have concluded that rainfall can predicted in future using the same method [88]. Haghizadeh et al., 2010, have proposed ANN model and Multiple Regression (MR) for prediction of total sediment at basin scale and they found that estimated rate of sediment yield by Artificial neural networks is much better fits with the observed data in comparison to MR model [89]. Subhajini and Raj, 2010, have put in a Computational Analysis of Optical Neural Network Models to Weather Forecasting; in this study they have compared Electronic Neural Network (ENN) model and optoelectronic neural network model. Overall their conclusion was, the training of opto-electronic neural network is fast compared to ANN. The accuracy of optoelectronic neural network is as good as ENN [90]. Durdu Omer Faruk, 2010, has experimented with A hybrid neural network and ARIMA model for water quality time series prediction. He has provided the results that the hybrid model provides much better accuracy over the ARIMA and neural network models for water quality predictions [91]. To identify and forecast the intensity of wind power and wind speed, Soman et al., 2010, have applied ANN and hybrid techniques over different time-scales, they found the accuracy in prediction associated with wind power and speed, based on numeric weather prediction (NWP) [92].

Forecasting daily rainfall at Mashhad Synoptic Station, Khalili et al., 2011, have applied Artificial Neural Networks model and they found that the black box model is capable of predicting the rainfall [93]. Pan et al., 2011, have experimented with feed forward neural network to predict Typhoon Rainfall. FNN is applied to estimate the residuals from the linear model to the differences between simulated rainfalls by a typhoon rainfall climatology model (TRCM) and observations and their results were satisfactory [94]. Joshi and Patel, 2011, have put in a review report on Rainfall-Runoff modeling using ANN, in the same study they have reviewed three neural network methods, Feed Forward Back Propagation (FFBP), Radial Basis Function (RBF) and Generalized Regression Neural Network (GRNN) and they have seen that GRNN flow estimation performances were close to those of the FFBP, RBF and MLR [95]. El-Shafie et al., 2011, have developed two rainfall prediction models i.e. Artificial Neural Network model and Multi regression model (MLR). An analysis of two statistical models developed for rainfall forecast on yearly and monthly basis in Alexandria, Egypt shows that an ANN has a better performance than an MLR model [96]. Rainfall forecasting in a mountainous region is a big task in itself Mekanik et al., 2011, have tried to do it using ANN modeling a feed forward ANN rainfall model was developed to investigate its potentials in forecasting rainfall. A monthly feed forward multi layer perceptron ANN rainfall forecasting model was developed for a station in the west mountainous region of Iran [97]. The temperature has a great effect in forecasting rainfall Amanpreet Kaur, and Harpreet Singh, 2011, have tested Artificial Neural Network in forecasting 
minimum temperature, they have used multi layer perceptron architecture to model the forecasting system and back propagation algorithm is used to train the network. They found that minimum temperature can be predicted with reasonable accuracy using ANN model [98]. El-Shafie et al., 2011, have proposed an idea of using adaptive neuro-fuzzy inference system based model for rainfall forecasting on monthly basis and they found that the ANFIS model showed higher rainfall forecasting accuracy and low error compared to the ANN model [99]. Tripathy et al., 2011, have experimented with ANN and Particle Swarm Optimization (PSO) Technique for weather forecasting and their experimental results indicate that the proposed approach is useful for weather forecasting [100]. As we have observed that many of the scientists have used ANN and various ANN models for forecasting Rainfall, Temperature, Wind and Flood etc., El-Shafie et al., 2011, have compared and studied Dynamic Vs Static neural network models for rainfall forecasting, they have developed AI based forecasting architectures using Multi-Layer Perceptron Neural Networks (MLPNN), RBFNN and Adaptive Neuron-Fuzzy Inference Systems (ANFIS), finally they concluded that the dynamic neural network namely IDNN could be suitable for modeling the temporal dimension of the rainfall pattern, thus, provides better forecasting accuracy [101]. Geetha and Selvaraj, 2011, have predicted Rainfall in Chennai using back propagation neural network model, by their research the mean monthly rainfall is predicted using ANN model. The model can perform well both in training and independent periods [102]. In various researches ANNs have been extensively used for simulation of rainfallrunoff and other hydrological processes. Reshma et al., 2011, have applied Artificial Neural Network for determination of Distributed Rainfall- Runoff Model Parameters and they found that the ANN technique can be successfully employed for the purpose of estimation of model parameters of distributed rainfall-runoff model [103]. Mohan Raju et al., 2011, have developed Artificial Neural-Network-Based Models for the Simulation of Spring Discharge, as their training and testing results revealed that the models were predicting the weekly spring discharge satisfactorily [104]. Predicting groundwater level is somehow a difficult task now a day because it varies place to place and round the globe, Mayilvaganan, and Naidu, 2011, have attempted to forecast groundwater level of a watershed using ANN and Fuzzy Logic. A three-layer feedforward ANN was developed using the sigmoid function and the back propagation algorithm. Now it has been observed that ANNs perform significantly better than Fuzzy Logic [105]. Elshafie et al., 2011, have tried to use neural network and regression technique for rainfall-runoff prediction finally they concluded that the results showed that the feed forward back propagation ANN can describe the behaviour of rainfall-runoff relation more accurately than the classical regression model [106]. In another research integrated artificial neural networkfuzzy logic-wavelet model is employed to predict Long term rainfall by Afshin et al., 2011. The results of the integrated model showed superior results when compared to the two year forecasts to predict the six-month and annual periods. As a result of the root mean squared error, predicting the two-year and annual periods is 6.22 and 7.11 , respectively. However, the predicted six months shows 13.15. [107]. Siou et al., 2011, have experimented with Complexity selection of a neural network model for flood forecasting, these models yield very good results, and the forecasted discharge values at the Lez spring are acceptable up to a 1-day forecasting horizon [108]. Saima et al., 2011, have reviewed on the various forecasting methods and hybrid models, they have put in a contradictory conclusion that there is no such model exists that can forecast accurately in all situations. This is because the distinct nature of the model [109].
At the end of literature survey from 1923 - 2012, In 2012, Sawaitul et al., have presented an approach for classification and prediction of future weather using back propagation algorithm, and discussed different models which were used in the past for weather forecasting, finally the study concludes that the new technology of wireless medium can be used for weather forecasting process. It also concludes that the Back Propagation Algorithm can also be applied on the weather forecasting data. Neural Networks are capable of modeling a weather forecast system [110].

\section{METHODOLOGY}

Two main architectures of ANN have been found by the above literature review and those are sufficiently suitable to predict chaotic behavior of monsoon rainfall are discussed in the following subsections-

\subsection{Radial Basis Function Network (RBFN)}

Lee et al., 1998, have studied that the spatial interpolation comparison 97 is concerned with predicting the daily rainfall at 367 locations based on the daily rainfall at nearby 100 locations in Switzerland. The whole region is divided into four sub-areas and each is modeled with a different method. Predictions in two larger areas were made by RBF networks based on the location information only. The two smaller areas were assumed to be implemented by the Orographic Effect which dictates that precipitation is proportional to elevation. Thus, predictions in these two areas were made using a simple linear regression model based on the elevation information only. They have concluded that the RBF networks produced good prediction while the linear models poor prediction [23].

After a study of RBFN, Chang et al., 2001, have found that RBFN is a suitable technique for a rainfall runoff model for three hours ahead floods forecasting [34]. He has proposed radial basis function (RBF) neural network $(\mathrm{NN})$ to develop a rainfallrunoff model for three-hour-ahead flood forecasting. The rainfall-runoff relation can be considered as a linear combination of some nonlinear RBFs. Rainfall and runoff events of the Lanyoung River collected during typhoons are used to train, validate, and test the network. The results show that the RBF NN can be considered as a suitable technique for predicting flood flow.

Maqsood et al., 2004 have proposed ensemble model performance is contrasted with multi-layered perceptron network (MLPN), Elman recurrent neural network (ERNN), RBFN, Hopfield model (HFM) predictive models and regression techniques. The data of temperature, wind speed and relative humidity are used to train and test the different models. With each model, 24-h-ahead forecasts are made for the winter, spring, summer and fall seasons. Moreover, the performance and reliability of the seven models are then evaluated by a number of statistical measures. Among the direct approaches employed, empirical results indicate that HFM is relatively less accurate and RBFN is relatively more reliable for the weather forecasting problem. In comparison, the ensemble of neural networks produced the most accurate forecasts. [42].

Exploring the new concept, soft computing models based on RBFN for 24-h weather forecasting, Maqsood et al., have applied a soft computing model based on a RBFN for 24-h weather forecasting of southern Saskatchewan, Canada. The model is trained and tested using hourly weather data of temperature, wind speed and relative humidity in 2001. The 
performance of the RBFN is compared with those of multilayered perceptron (MLP) network, Elman recurrent neural network (ERNN) and Hopfield model (HFM) to examine their applicability for weather analysis. Reliabilities of the models are then evaluated by a number of statistical measures. The results indicate that the RBFN produces the most accurate forecasts compared to the MLP, ERNN and HFM. They have concluded that the RBFN produces the most accurate forecasts compared to the MLP, ERNN and HFM [54].

Patil and Ghatol, 2010, have revealed that the flapping of butterfly wings causing a tropical cyclone. Numerous researcher have introduces number of models for rainfall forecasting. No two model forecast the same situation in exactly same way. On the same time no single model is reliable for rainfall forecasting. Climate and rainfall are highly non-linear phenomena in nature exhibiting what is known as the "butterfly effect". They have used various ANN topologies such as radial basis functions and multilayer perceptron with Levenberg Marquardt and momentum learning rules for predicting rainfall using local parameters and they found the topologies fit for the same task [85].

Joshi and Patel, 2011, have put in a review report on RainfallRunoff modeling using ANN, in the same study they have reviewed three neural network methods, Feed Forward Back Propagation (FFBP), Radial Basis Function (RBF) and Generalized Regression Neural Network (GRNN) and they have seen that GRNN flow estimation performances were close to those of the FFBP, RBF and MLR. The theoretical basis of the $\mathrm{RBF}$ approach lies in the field of interpolation of multivariate functions. The solution of exact interpolating RBF mapping passes through every data point. Different number of hidden layer neurons \& spread constants were tried in study [95].

As we have observed that many of the scientists have used ANN and various ANN models for forecasting Rainfall, Temperature, Wind and Flood etc., El-Shafie et al., 2011, have compared and studied Dynamic Vs Static neural network models for rainfall forecasting, they have developed AI based forecasting architectures using Multi-Layer Perceptron Neural Networks (MLPNN), RBFNN and Adaptive Neuron-Fuzzy Inference Systems (ANFIS), finally they concluded that the dynamic neural network namely IDNN could be suitable for modeling the temporal dimension of the rainfall pattern, thus, provides better forecasting accuracy [101].

\subsection{Back-Propagation Network (BPN)}

Lekkas et al., 2004 have used a multilayer back propagation network and found that BPN will not always find the correct weight and biases for the optimum solution, whereas their results supported the hypothesis that ANNs can produce qualitative forecast. A 7 hour ahead forecast in particular proves to be of fairly high precision, especially when an error prediction technique is introduced to the ANN model. In real-time applications, like flow regulation and flood forecasting where the precision and modeling speed are crucial, black box models and signal processing techniques need to be implemented. As is presented by this case study, error prediction manages to correct the model output and produce an excellent forecast. [44].

In coastal areas it is an enormous prediction of tidal level, Tsong-Lin Lee, 2004, has predicted long-term tidal level using BPN network, as compare to conventional harmonic method, he concluded that back-propagation neural network mode also efficiently predicts the long-term tidal levels. The main constituents of tide in the conventional harmonic method require a large number of observed tidal data using the spectral method based on two years of data. In addition, the parameters in the harmonic tide level model are estimated by the method of least squares for a long duration (more than one month). The BPN method only requires a part of observed tidal data to determine the total number of constituent tides and the harmonic parameters. The results of the case study show that the major constituents can be obtained by using two-month measured data. Comparing the BPN model results and the harmonic data, BPN for predicting one-year tidal level can be satisfactorily achieved with 15 days of observed data. [49].

On the basis of humidity, dew point and pressure in India, Enireddy et al., 2010, have used the back propagation neural network model for predicting the rainfall. The back propagation neural network model is used for predicting the rainfall based on humidity, dew point and pressure in the country INDIA. TwoThird of the data was used for training and One-third for testing. The number of training and testing patterns is 250 training and 120 testing. In the training they have obtained $99.79 \%$ of accuracy and $94.28 \%$ in testing. For rainfall prediction, Artificial Neural Network was applied and the rainfall was predicted in India. According to the results back propagation neural network were acceptably accurate and can be used for predicting the rainfall. So by using this method for prediction we can find the amount of rainfall in the region by using the attributes like humidity, dew point and pressure. From these results they have concluded that rainfall can predicted in future using the same method [88].

ANN approach is extensively used in the water resources literature. In most of the studies of ANN either the conventional BPN or the RBFN was employed to train the neural networks. Joshi and Patel, 2011, have put in a review report on RainfallRunoff modeling using ANN, in the same study they have reviewed three neural network methods, BPN, RBFN and GRNN and they have seen that GRNN flow estimation performances were close to those of the FFBP, RBF and MLR. An important result of the study is the positive contribution of the utilization of initial statistical analysis results in determining the ANN input layer node number [95].

The temperature has a great effect in forecasting rainfall Kaur and Singh, 2011, have tested ANN in forecasting minimum temperature, they have used multi layer perceptron architecture to model the forecasting system and back propagation algorithm is used to train the network. Neural networks have been trained to perform complex functions in various fields of application including forecasting, pattern recognition, identification, classification and control systems. From this work it can be concluded that a feed-forward ANN model using backpropagation algorithm is developed to identify the minimum temperature. The results show that an appropriate accuracy can be achieved using this network. The neural network approach for weather forecasting is capable of yielding good results and can be considered as an alternative to traditional meteorological approaches. Further, this approach is also able to determine the values of other parameters like maximum relative humidity, minimum relative humidity, maximum temperature etc in a particular year. They found that minimum temperature can be predicted with reasonable accuracy using ANN model [98].

Since the mechanisms of rainfall are still not understood well, ANNs are a good choice worth trying to analyze the relationship between meteorological parameters and rainfall. A real world case study was observed in Chennai for 32 years of monthly mean data with meteorological parameters such as wind speed, 
mean temperature, relative humidity, aerosol values (RSPM) in the area were used to develop the ANN model. In order to forecast rainfall in Chennai City, BPNs, a data driven technique based on the working principle of biological neurons are applied in this study. Geetha and Selvaraj, 2011, have predicted Rainfall in Chennai using back propagation neural network model, by their research the mean monthly rainfall is predicted using ANN model. They have observed that the model can perform well both in training and independent periods [102].

Predicting groundwater level is somehow a difficult task now a day because it varies place to place and round the globe, Mayilvaganan, and Naidu, 2011, have attempted to forecast groundwater level of a watershed using ANN and Fuzzy Logic. A three-layer feed-forward ANN was developed using the sigmoid function and the back propagation algorithm. Now it has been observed that ANNs perform significantly better than Fuzzy Logic [105].

El-shafie et al., 2011, have tried to use neural network and regression technique for rainfall-runoff prediction finally they concluded that the results showed that the BPN can describe the behaviour of rainfall-runoff relation more accurately than the classical regression model [106].

Sawaitul et al., 2011, have presented an approach for classification and prediction of future weather using back propagation algorithm, and discussed different models which were used in the past for weather forecasting, finally the study concludes that the new technology of wireless medium can be used for weather forecasting process. It also concludes that the BPN Algorithm can also be applied on the weather forecasting data. Neural Networks are capable of modeling a weather forecast system [110].

\subsection{Other Methods}

After development of recurrent Sigma- Pi neural network rainfall forecasting system, Chow and Cho, 1997, have concluded that the neural network based now casting system is capable of providing a reliable rainfall now casting in Hong Kong [22]. Aliev et al., 2008, have proposed, fuzzy recurrent neural network (FRNN) based time series forecasting method for solving forecasting problems, in an experiment and they found that the performance of the proposed method for forecasting fuzzy time series shows its high efficiency and effectiveness for a wide domain of application areas ranging from weather forecasting to planning in economics and business [67]. In 2001 Luk et al., have developed and compared three types of ANNs suitable for rainfall prediction i.e. multilayer feed forward neural network, Elman partial recurrent neural network and time delay neural network [32]. For a rainfall runoff relationship Harun and Irwan, 2002, have concluded that the performance of neural network model is better than HEC-HMS and MLR models for modeling the rainfall runoff relationship [37]. In 2004 Maqsood et al., have found that HFM is relatively less accurate and RBFN is relatively more reliable for the weather forecasting problems and in comparison the ensembles of neural networks produced the most accurate forecast [42].

\section{RESULTS}

After review of a wide range of ANN architectures for rainfall forecasting, it has been observed that most of the researchers have used BPN and RBFN techniques for forecasting various weather phenomenon e.g. rainfall, temperature, flood, rainfall- runoff etc, and found significant results using the same architectures. Hence in literature review of moreover 25 years of researches. Most of the scientists have concluded that BPNN and RBFN are the appropriate method to predict weather phenomenon. In the development of Recurrent Sigma-pi Neural Network Rainfall Forecasting System in Hong Kong by Chow and Cho, 1997, it has been observed that A Recurrent Sigma-Pi neural network was selected as the network architecture providing strong dynamical properties for the modeling of this type of non-linear time series. The overall forecasting relative percentage error is about $3-5 \%$, the results indicate that the neural network-based now casting system is capable of providing a reliable rainfall now casting in Hong [22]. Lee et al., 1998, have applied the "divide-and-conquer" approach to divide the whole region into four areas and the problem was solved separately based on the following assumptions: first, rainfall has a different pattern in different areas; second, rainfall pattern within smaller areas is continuous and smooth; third, orographic effect exists. Predictions of the two large areas using RBF networks were reasonably good. But, for the two small areas, predictions were not good since the orographic effect was not apparent [23]. In a comparative study of various ANN architectures such as, MLP, ERNN, HFM and RBFN, performed by Maqsood et al., 2004 have strongly favoured that the performance of RBFN is more promising and accurate than other three methods [42]. For prediction of tidal level in coastal areas Tsong-Lin Lee, 2004, has tried BPN technique and found to be an appropriate solution as compare to other conventional harmonic methods [49]. An innovative application of ANN for weather forecasting in space is applied by Jon Vandegriff et al., 2005, and they have found that an average of the absolute error using this approach is about 14.5 for both training and test data sets. The network consistently beats this simple forecast, and so ANN provides better accuracy than existing methods for forecasting shock arrivals in the space weather [52]. Ozgur KISI, 2005, has selected three simple neural network architectures, i.e. ANN, Auto-Regressive Models and sum of square errors, for comparison of forecasting probabilities and he found that NNs were able to produce better results than AR models when given the same data inputs. The potential of ANN models for simulating the hydrologic behavior of stream flow has been presented in this study. The greatest difficulty lay in determining the appropriate model inputs for such a problem. The results obtained with ANNs for 1-day ahead forecasts are better than those reached in the AR models and confirm the ability of this approach to provide a useful tool in solving a specific problem in hydrology, that of stream flow forecasting. The results suggest that the ANN approach may provide a superior alternative to the AR models for developing input \{output simulations and forecasting models in situations that do not require modeling of the internal structure of the watershed [53]. In 2006, Somvanshi et al., have proved that ANN model can be used as an appropriate forecasting tool to predict the rainfall, which out performs the ARIMA model. Complexity of the nature of annual rainfall record has been studied using the ANN and ARIMA techniques. An annual rainfall data spanning over a period of 1901- 2003 of Hyderabad region was used to develop and test the models. Autocorrelation and partial autocorrelation coefficient for various lags (in year) of rainfall data was used to find out number of past observations as inputs to neural network. The present analysis uses four past observations as inputs to neural network model. The study reveals that ANN model can be used as an appropriate forecasting tool to predict the rainfall, which out performs the ARIMA model. Further refinement of the model using the data separately from the different zones of the country may be useful for the long-term prediction [55]. Using ANNs and Fuzzy Logic, Mayilvaganan, and Naidu, 2011, 
have tried to predict ground water level and they have concluded that ANN performs better than Fuzzy Logic [105]. It has been also proved by the contribution of Karmakar et al., 2008, 2009, 2010 , that BPN in deterministic as well as parametric forecast are more efficient technique over the statistical model for forecasting long-range monsoon rainfall over the high resolution geographical region such as district or sub-division level [111114]. He has successfully obtained global minima up to the level of $10^{-04}$ during the training period. And also has obtained more than $80 \%$ accuracy in prediction during the independent period.

To predict long-range TMRF (in mm.) over the smaller scale geographical region "district", eight parameters BPN model has been developed [47]. Eight parameters BPN model is depicted in the following Fig. 1 Wherein, eight input parameters which are physically connected with TMRF over this region are used to input in the BPN. Three neurons in hidden layer, one neuron in output layer, a total of $8.3+3=27$ trainable weights including 3 biases in hidden layer and 1 bias in output layer have been used to train the model and observe TMRF as model output. It is observed that three neurons can be utilize in hidden layer therefore three neurons in hidden layer is chosen. The detail discussion of selection of neurons in hidden layer is given in following subsection. The neuron output is obtained as $f\left(x_{\mathrm{j}}\right)$. Where $f$ is a transfer function typically the sigmoid function. The skeleton of the model is shown in Fig. 2 and Fig. 3.

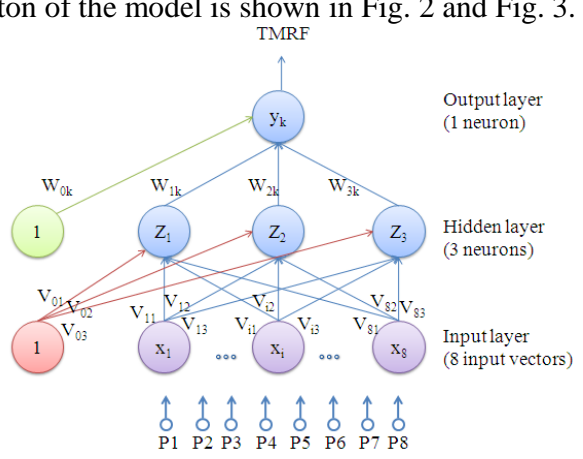

Fig. 1. 8-Parameters BPN Model

The initial random trainable weights including biases on hidden and output layer as shown in Fig. 1 is selected randomly by using "Random" class of Java is optimized by exercising 20 , 00000 epochs. The optimized weights are shown in Table 1 . The trained network presented excellent learning curve started with local minima MSE $=0.0010583407253476$ to global minima MSE $=6.555600546440187 \mathrm{E}-04$ that is exceptionally close to the optimum value of 0 as shown in the Fig. 2.

\begin{tabular}{|c|c|}
\hline 1.0000000000000000 & $0 ! 18447$ \\
\hline 0.1000000000000000 & ere \\
\hline 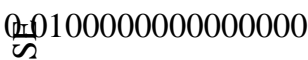 & $\begin{array}{l}182 \\
98675\end{array}$ \\
\hline 8.0010000000000000 & \\
\hline 0.0001000000000000 & $\begin{array}{l}39887343^{2}-04 \\
\text { Epochs }\end{array}$ \\
\hline
\end{tabular}

Fig. 2 Minimizing error (MSE) during training process BPN model in parametric forecast has been developed and evaluated. The performance of the model during the training period and independent/testing period is given in the Table. 1 and Fig. 1 respectively.

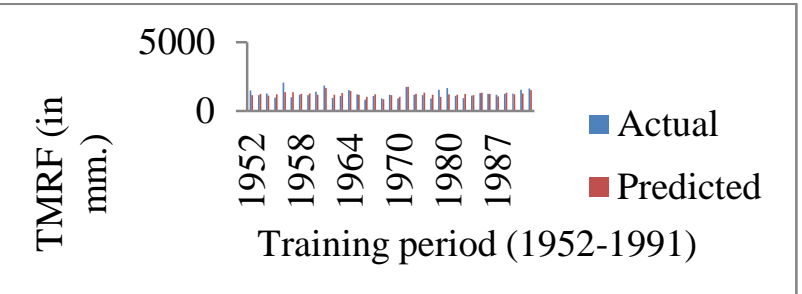

Fig. 3. Performance of BPN in deterministic forecast during the training period (1951-1991)

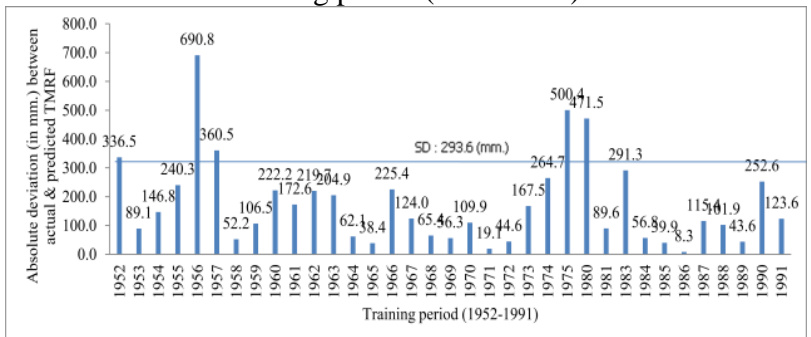

Fig. 4. Absolute deviation between actual TMRF and predicted TMRF during training period (1951-1991)

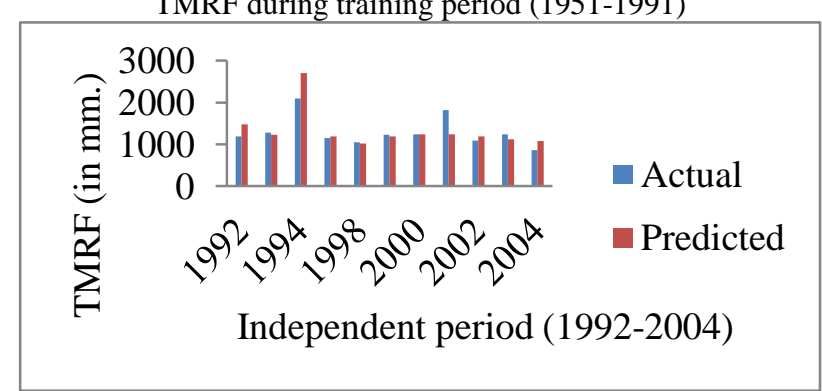

Fig. 5. Performance of BPN in deterministic forecast during the independent/testing period (1992-2004)

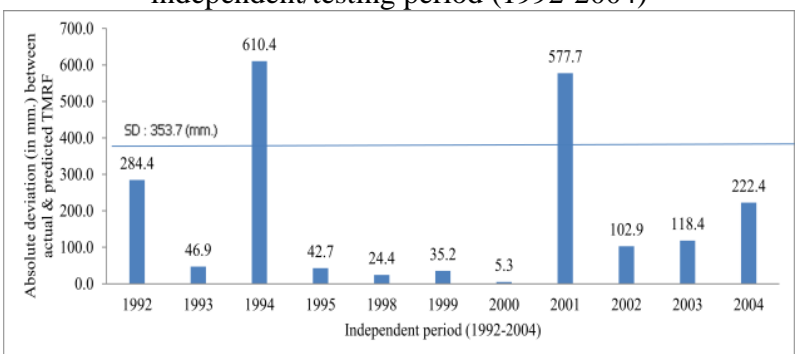

Fig. 6. Absolute deviation between actual TMRF and predicted TMFR during independent period/testing period

During an intense study of applications of various architectures of ANN, it has been found that the BPN and RBFN are the methods which have been used by most of the researchers and the result of their experiment found to be satisfactory without any scientific controversy. In general it has been observed that out of various forecasting techniques such as statistical and numerical modeling, over the meteorological data, ANN is proved to be an appropriate technique undoubtedly for forecasting various weather phenomenons.

\section{CONCLUSION}

This study concentrates on capabilities of ANN in prediction of several weather phenomenon such as rainfall, temperature, flood and tidal level etc. finally it has been concluded that the major architectures i.e. BPN, RBFN, MLP are sufficiently suitable to predict weather phenomenon. In the comparative study among various ANN techniques, BPN and RBFN are found as appropriate solutions for prediction of long-range weather forecasting. The study of BPN and RBFN for long range 
meteorological parameters pattern recognition over smaller scale geographical region shows a good performance and reasonable prediction accuracy. However, some shortcoming also has been found. These models are exceptionally slow during the learning process. Sometime training process of these models may introduce a temporal nervousness to the user.

\section{ACKNOWLEDGMENTS}

We are thankful to Bhilai Institute of Technology (BIT), Durg, Chhattisgarh, India for providing sufficient paraphernalia, facility as well as encouragement.

\section{REFERENCES}

[1]. Walker, G. T., 1923, "Correlation in Seasonal Variations of Weather, III. A Preliminary Study of World Weather". Mem. India Meteorol. Dep., XXIV, 75-131.

[2]. Walker, G. T., 1924, "Correlation in Seasonal Variations of Weather, IV. A Further Study of World Weather". Mem. India Meteorol. Dep., XXIV, 275- 332.

[3]. Gowariker, V., Thapliyal, V., Sarker, R. P., Mandal, G. S. and Sikka, D. R., 1989, "Parametric and Power Regression Models: New Approach to Long Range Forecasting of Monsoon Rainfall in India”. Mausam, 40, 115- 122.

[4]. Gowariker, V., Thapliyal, V., Kulshrestha, S. M., Mandal, G. S., Sen Roy, N., and Sikka, D. R., 1991, "A Power Regression Model for Long Range Forecast of Southwest Monsoon Rainfall over India”. Mausam, 42, 125-130.

[5]. Thapliyal, V., and Kulshrestha, S. M., 1992, "Recent Models for Long Range Forecasting of Southwest Monsoon Rainfall over India”. Mausam, 43, 239-248.

[6]. Thapliyal, V., 1997, "Preliminary and Final Long Range Forecasts for Seasonal Monsoon Rainfall over India". J. Arid Environ., 36, 385-403.

[7]. Rajeevan, M., Guhathakurta, P. and Thapliyal, V., 2000, "New Models for Long Range Forecasting of Monsoon Rainfall over Northwest and Peninsular India". Meteorol. Atmos. Phys., 73, 211-225.

[8]. Rajeevan M., 2001, "Prediction of Indian Summer Monsoon: Status, Problems and Prospects". Current Science, 81, 1451-1457.

[9]. Thapliyal, V., and Rajeevan, M., 2003, "Updated Operational Models for Long-Range Forecasts of Indian Summer Monsoon Rainfall”. Mausam, 54, 495-504.

[10]. Rajeevan, M., Pai, D.S., Dikshit, S.K., and Kelkar, R. $R ., 2004$, "IMD's New Operational Models for Long-Range Forecast of Southwest Monsoon Rainfall over India and Their Verification for 2003", Current Science, 86 (3), 422431.

[11]. Guhathakurta, P., 2000, "New Models for Long Range Forecasts of Summer Monsoon Rainfall over North West and Peninsular India", Meteor. \& Atomos. Phys.,73 (3), 211-255.

[12]. Guhathakurta, P., Rajeevan, M., and Thapliyal, V., 1999, "Long Range Forecasting Indian Summer Monsoon Rainfall by Hybrid Principal Component Neural Network Model", Meteorology and Atmospheric Physics, SpringerVerlag, Austria.
[13]. Parthasarathy, B., Rupa Kumar, K., and Munot, A. A., 1991, "Evidence of Secular Variations in Indian Summer Monsoon Rainfall Circulation Relationships". J. Climate, 4, 927-938.

[14]. Hastenrath, S., and Greischar, L., 1993, "Changing Predictability of Indian Monsoon Rainfall Anomalies". Proc. Indian Acad. Sci. (Earth Planet. Sci.), 102, 35-47.

[15]. Guhathakurta P., (2006), "Long-Range Monsoon Rainfall Prediction of 2005 for the Districts and SubDivision Kerala with Artificial Neural Network", Current Science, 90(6), pp-773-779.

[16]. Krishnamurthy, V., and Kinter, J. L., 2002, “The Indian Monsoon and its Relation to Global Climate Variability". Global Climate - Current Research and Uncertainties in the Climate System (eds Rodo, X. and Comin, F. A.), 186-236.

[17].Krishnamurthy, V., and Kirtman, B. P., 2003, "Variability of the Indian Ocean: Relation to Monsoon and ENSO". Q. J. R.” Meteorol. Soc., 129, 1623-1646

[18]. Sahai, A. K., Grimm, A. M., Satyan. V. and Pant, G. B. 2002, "Prospects of Prediction of Indian Summer Monsoon Rainfall using Global SST Anomalies". IITM Research Report No. RR-093.

[19]. Guhathakurta, P., 1999, "Long Range Forecasting Indian Summer Monsoon Rainfall By Principle Component Neural Network Model", Meteor. \& Atomos. Phys., 71, 255-266.

[20]. Guhathakurta, P., 1998, "A Hybrid Neural Network Model for Long Range Prediction of All India Summer Monsoon Rainfall", Proceedings of WMO international workshop on dynamical extended range forecasting, Toulouse, France, November 17-21, 1997, PWPR No. 11, WMO/TD. 881, pp 157-161.

[21]. Basu Sujit \& Andharia H I, 1992, "The Chaotic time series of Indian Monsoon rainfall and its prediction", Proc. Indian Acad. Sci., Vol. 101, No. 1, pp 27-34.

[22]. Chow T. W. S. and Cho S. Y., 1997, "Development of a Recurrent Sigma-Pi Neural Network Rainfall Forecasting System in Hong Kong”, Springer-Verlag, pp. 66-75.

[23]. Lee Sunyoung, Cho Sungzoon, Wong Patrick M., 1998, "Rainfall Prediction Using Artificial Neural Networks", Journal of Geographic Information and Decision Analysis, vol. 2, no. 2, pp. $233-242$.

[24]. Hsieh, W.H., and Tang, B., 1998, "Applying Neural Network Models to Prediction and Analysis in Meteorology and Oceanography”. Bull. Amer. Met. Soc., 79, 855-1870.

[25]. Dawson Christian W., Wilby Robert, "An artificial neural network approach to rainfall runoff modeling", 1998, Hydrological Sciences-Journal, 43(1), pp. 47-66.

[26]. Guhathakurtal P., Rajeevan2 M., and Thapliyal2 V., 1999, "Long Range Forecasting Indian Summer Monsoon Rainfall by a Hybrid Principal Component Neural Network Model", Meteorol. Atmos. Phys., 71, pp. 255-266.

[27].Ricardo, M., Trigo, Jean, P., Palutikof, 1999, "Simulation of Daily Temperatures for Climate Change Scenarios over Portugal: A Neural Network Model Approach", University of East Anglia, Norwich, NR4 7TJ, United Kingdom, climate research (Clim., Res.), 13, 45-59, 1999. 
[28]. Jones, C., Peterson, P., 1999, "A New Method for Deriving Ocean Surface Specific Humidity and Air Temperature: An Artificial Neural Network Approach", J. Applied Meteorology, American Meteorological Society, $38,1229-1245$.

[29].Guhathakurta, P., 1999, "A Short Term Prediction Model for Surface Ozone At Pune: Neural Network Approach", Vayu mandal, Special issue on Asian monsoon and pollution over the monsoon environment, 29(1-4), 355-358.

[30]. Guhathakurta, P., 1999, "A Neural Network Model for Short Term Prediction of Surface Ozone at Pune", Mausam, 50(1), 91-98.

[31]. Toth E. *, Brath A., Montanari A., 2000, "Comparison of short-term rainfall prediction models for real-time flood forecasting", Journal of Hydrology, Elsevier, 239, pp. 132147.

[32]. Luk Kin C, Ball J. E. and Sharma A., 2001, “An Application of Artificial Neural Networks for Rainfall Forecasting", 33, pp. 883-699.

[33]. Michaelides Silas Chr *, Pattichis Constantinos S. and Kleovouloub Georgia, 2001, "Classification of rainfall variability by using artificial neural networks", International Journal Of Climatology, pp. 1401-1414.

[34]. Chang Fi-John, Liang Jin-Ming, and Chen YenChang,2001, "Flood Forecasting Using Radial Basis Function Neural Networks", IEEE Transactions On Systems, Man, And Cybernetics, vol. 31, no. 4, pp. 530535 .

[35]. Brath A., Montanari A. and Toth E., 2001, "Neural networks and non-parametric methods for improving ealtime flood forecasting through conceptual hydrological models", Hydrology and Earth System Sciences, 6(4), pp. 627-640.

[36]. Rajurkar M. P., Kothyari U. C., Chaube U. C., 2002, "Artificial neural networks for daily rainfall-runoff modelling", Hydrologkal Sciences-Journals, 47(6), pp. 865877.

[37]. Harun Sobri, Nor Irwan Ahmat \& Mohd. Kassim Amir Hashim, 2002, "Artificial neural network model for rainfallrunoff relationship", Journal Technology, Malaysia, pp. 112.

[38].Iseri, Y. G. C., Dandy, R., Maier, A., Kawamura, and Jinno, K., 2002, "Medium Term Forecasting of Rainfall Using Artificial Neural Networks", Part 1 background and methodology, Journal of Hydrology , 301 (1-4), 1834-1840.

[39]. Silva, A. P., 2003, "Neural Networks Application to Spatial Interpolation of Climate Variables", Carried Out By, STSM on the Framework of COST 719 ZAMG, Vienna 610 October 2003

[40].Snell., Seth, E., Gopal, Sucharita, Kaufmann, Robert, K., 2003, "Spatial Interpolation of Surface Air Temperatures Using Artificial Neural Networks: Evaluating Their Use for Downscaling GCMs", Journal of Climate, 13 (5), 886-895.

[41].Blender, R., 2003, "Predictability Study of the Observed and Simulated European Climate using Linear Regression", Q. J. R. Meteorol. Soc. (2003), 129, 2299-2313.

[42]. Maqsood Imran, Khan Muhammad Riaz, Abraham Ajith, 2004, Neural Comput \& Applic, 13, pp. 112-122.
[43]. Pasero Eros, Moniaci Walter, 2004, "Artificial Neural Networks for Meteorological Nowcast", IEEE international Conference,

[44]. Lekkas D.F.,* Onof1 C., Lee1 M. J., Baltas2 E.A., 2004, "Application of artificial neural networks for flood forecasting”, Global Nest, Vol 6, No 3, pp 205-211.

[45]. Shu Chang and Burn Donald H., 2004, "Artificial neural network ensembles and their application in pooled flood frequency analysis", Water Resources Research, vol. 40.

[46]. Nayaka P.C., *, Sudheerb K.P.,1Ranganc, D.M.,2, Ramasastrid K.S.,3, 2004, "A neuro-fuzzy computing technique for modeling hydrological time series", Journal of Hydrology, Elsevier, 291, pp. 52-66.

[47]. $\quad W u$ Jy S., P.E., ASCE1 M.; Han2 Jun; Annambhotla3 Shastri; and Scott Bryant4, 2004, "Artificial Neural Networks for Forecasting Watershed Runoff and Stream Flows", Journal Of Hydrologic Engineering, 216

[48]. Abdel-Aal R.E. *, 2004, "Hourly temperature forecasting using abductive networks", Elsevier, 17, pp. 543-556.

[49]. Lee Tsong-Lin, 2004, "Back-propagation neural network for long-term tidal predictions", Elsevier, 31, pp. $225-238$.

[50]. Chaudhuri Sutapa, Chattopadhyay Surajit, 2005 , "Neuro-computing based short range prediction of some meteorological parameters during the pre-monsoon season", Springer-Verlag, 9, pp. 349-354

[51]. Lin Gwo-Fong * and Chen Lu-Hsien, 2005 , "Application of an artificial neural network to typhoon rainfall forecasting”, Hydrological Processes, 19, pp. 1825 1837.

[52]. Vandegriff Jon *, Wagstaff Kiri, George Ho, Plauger Janice, 2005, "Forecasting space weather: Predicting interplanetary shocks using neural networks", Elsevier, vol 36, pp. 2323-2327.

[53]. KISI Ozgur, 2005, "Daily River Flow Forecasting Using Artificial Neural Networks and Auto-Regressive Models", Turkish J. Eng. Env. Sci., vol 29, pp. 9 - 20.

[54]. Maqsood Imran, Khan Muhammad Riaz, Huang Guo H., Abdalla Rifaat, 2005, “Application of soft computing models to hourly weather analysis in southern Saskatchewan, Canada", Elsevier, vol 18, pp. 115-125.

[55]. Somvanshi V.K., Pandey O.P., Agrawal P.K. Kalanker1 N.V., Prakash M.Ravi and Chand Ramesh, 2006, "Modelling and prediction of rainfall using artificial neural network and ARIMA techniques", J. Ind. Geophys. Union, Vol.10, No.2, pp.141-151.

[56]. Srikalra Niravesh and Tanprasert Chularat, 2006 "Rainfall Prediction for Chao Phraya River using Neural Networks with Online Data Collection", Malaysia, pp. 1315 .

[57]. Kumarasiri A.D.and Sonnadara D.U.J., 2006 , "Rainfall Forecasting: An Artificial Neural Network Approach", Proceedings of the Technical Sessions, vol 22, pp. 1-13.

[58]. Kumar D. Nagesh, Reddy M. Janga and Maity Rajib, 2006, "Regional Rainfall Forecasting using Large Scale 
Climate Teleconnections and Artificial Intelligence Techniques", Journal of Intelligent Systems, Vol. 16, No.4, pp. 307-322.

[59].Guhathakurta, P., 2006, "Long-Range Monsoon Rainfall Prediction of 2005 for the Districts and Sub-Division Kerala With Artificial Neural Network", Current Science, 90 (6), pp-773-779.

[60]. Bustami Rosmina, l BessaihNabi, Charles Bong, Suhaila Suhaili, 2007, "Artificial Neural Network for Precipitation and Water Level Predictions of Bedup River", International Journal of Computer Science, vol 34:2.

[61]. Paras, Mathur Sanjay, Kumar Avinash, and Chandra Mahesh, 2007, "A Feature Based Neural Network Model for Weather Forecasting", World Academy of Science, Engineering and Technology, vol 34, pp. 66-73.

[62]. Hayati Mohsen, and Mohebi Zahra, 2007, "Application of Artificial Neural Networks for Temperature Forecasting", World Academy of Science, Engineering and Technology, vol 28, pp. 275-279.

[63]. Morid Saeid, Smakhtin Vladimir and Bagherzadeh K., 2007, "Drought forecasting using artificial neural networks and time series of drought indices", Royal Meteorological Society, vol. 27, pp. 2103-2111.

[64]. Hayati Mohsen, and Shirvany Yazdan, 2007, "Artificial Neural Network Approach for Short Term Load Forecasting for Illam Region", World Academy of Science, Engineering and Technology, vol. 28, pp. 280-284.

[65]. Lucio P. S., Conde F. C., Cavalcanti I. F. A., Serrano A. I., Ramos A. M., and Cardoso A. O., 2007, "Spatiotemporal monthly rainfall reconstruction via artificial neural network - case study: south of Brazil", Advances in Geosciences, vol. 10, pp. 67-76.

[66]. Hartmann, Heikea * Becker Stefanb and Kinga Lorenz, 2007, "Predicting summer rainfall in the Yangtze River basin with neural networks", Royal Meteorological Society.

[67]. Aliev R. A., Fazlollahi B., Aliev R. R., Guirimov B., 2008, "Linguistic time series forecasting using fuzzy recurrent neural network", Soft Comput, vol. 12, pp. 183190

[68]. Chattopadhyay Surajit and Chattopadhyay Goutami, 2008, "Identification of the best hidden layer size for three layered neural net in predicting monsoon rainfall in India", Journal of Hydroinformatics, vol. 10(2), pp. 181-188.

[69]. Hung N. Q., Babel M. S., Weesakul S., and Tripathi N. $K ., 2008$, "An artificial neural network model for rainfall forecasting in Bangkok, Thailand", Hydrology and Earth System Sciences, vol. 5, pp. 183-218.

[70]. Aytek Ali, Asce $M$ and Alp Murat, 2008, “An application of artificial intelligence for rainfall-runoff modeling”, J. Earth Syst. Sci., vol. 117, No. 2, pp. 145-155.

[71]. Chattopadhyay Goutami, Chattopadhyay Surajit, Jain Rajni, 2008, "Multivariate forecast of winter monsoon rainfall in India using SST anomaly as a predictor: Neurocomputing and statistical approaches",

[72]. Win Khaing Mar and Thu Naing Thinn, 2008 , "Optimum Neural Network Architecture for Precipitation Prediction of Myanmar", World Academy of Science, Engineering and Technology, vol. 48, pp. 130-134.
[73]. Karmakar Sanjeev et al., 2008, "Development of a Satellite Based Agriculture Meteorological Information System (AGRIMETCast) in the Context of Remote Places of Chhattisgarh Using Remote Sensing Data" , India Society of Remote Sencing, Ahmedabad, India., SAC (ISRO)

[74]. Karmakar Sanjeev et al., 2008, "Development of Artificial Neural Network Models for Long-Range Meteorological Parameters Pattern Recognition over the Smaller Scale Geographical Region", IEEE Computer Society, Washington, DC, USA., 8-10 Dec. 2008, pp.1 - 6.

[75]. Hocaoglu Fatih O., Oysal Yusuf, Kurban Mehmet, 2009, "Missing wind data forecasting with adaptive neuro-fuzzy inference system", Springer-Verlag London, vol. 18, pp. 207-212.

[76]. Solaimani Karim, 2009, "Rainfall-runoff Prediction Based on Artificial Neural Network (A Case Study: Jarahi Watershed)", American-Eurasian J. Agric. \& Environ. Sci., vol. 5(6), pp. 856-865.

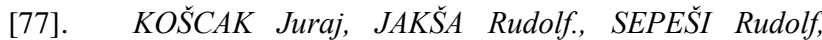
SINCÁK Peter., 2009, "Weather forecast using Neural Networks", 9th Scientific Conference of Young Researchers

[78]. Karamouz M.; . Fallahi M, Nazif S. and Farahani M. Rahimi, 2009, "Long Lead Rainfall Prediction Using Statistical Downscaling and Arti cial Neural Network Modeling", Transaction A: Civil Engineering, Vol. 16, No. 2, pp. 165-172.

79]. Widjanarko Bambang Otok, Suhartono, 2009, "Development of Rainfall Forecasting Model in Indonesia by using ASTAR, Transfer Function, and ARIMA Methods", European Journal of Scientific Research, Vol.38 No.3, pp.386-395.

[80]. Nekoukar Vahab, Taghi Mohammad, Beheshti Hamidi, 2010, "A local linear radial basis function neural network for financial time-series forecasting", Springer Science, vol. 23 , pp. 352-356.

[81]. Weerasinghe H.D.P.,. Premaratne H.L and Sonnadara D.U.J., 2010, "Performance of neural networks in forecasting daily precipitation using multiple sources", J.Natn.Sci.Foundation Sri Lanka, vol. 38(3), pp. 163-170.

[82]. Luenam Pramote, Ingsriswang Supawadee, Ingsrisawang Lily, Aungsuratana Prasert, and Khantiyanan Warawut, 2010, "A Neuro-Fuzzy Approach for Daily Rainfall Prediction over the Central Region of Thailand", ISSN 2010, vol. 1.

[83]. Wu C. L., Chaul K. W, and Fan C., 2010, "Prediction of Rainfall Time Series Using Modular Artificial Neural Networks Coupled with Data Preprocessing Techniques", Journal of Hydrology, Vol. 389, No. 1-2, pp. 146-167.

[84]. Nastos Panagiotis, Moustris Kostas, Larissi Ioanna and Paliatsos Athanasios, 2010, "Rain intensity forecast using Artificial Neural Networks in Athens, Greece", Geophysical Research Abstracts, Vol. 12.

[85]. Patil C. Y. and Ghatol A. A., 2010, "Rainfall forecasting using local parameters over a meteorological station: an artificial neural network approach", International J. of Engg. Research \& Indu. Appls, Vol.3, No. II, pp 341356. 
[86]. Tiron Gina, and Gosav Steluţa, 2010, "The july 2008 rainfall estimation from BARNOVA WSR-98 D Radar using artificial neural network", Romanian Reports in Physics, Vol. 62, No. 2, pp. 405-413.

[87]. Goyal Manish Kumar, Ojha Chandra Shekhar Prasad, 2010, "Analysis of Mean Monthly Rainfall Runoff Data of Indian Catchments Using Dimensionless Variables by Neural Network", Journal of Environmental Protection, vol. 1, pp. 155-171.

[88]. . . Vamsidhar Enireddy Varma K.V.S.R.P..Sankara Rao P satapati Ravikanth, 2010, "Prediction of Rainfall Using Backpropagation Neural Network Model", International Journal on Computer Science and Engineering, Vol. 02, No. 04, pp. 1119-1121.

[89]. Haghizadeh Ali, Teang shui Lee, Goudarzi Ehsan, 2010, "Estimation of Yield Sediment Using Artificial Neural Network at Basin Scale", Australian Journal of Basic and Applied Sciences, vol. 4(7), pp. 1668-1675.

[90]. Subhajini A . C. and Joseph Raj V., 2010, "Computational Analysis of Optical Neural Network Models to Weather Forecasting", International Journal of Computer Science Issue, Vol.7, Issue 5, pp. 327-330.

[91]. Omer Faruk Durdu, 2010, "A hybrid neural network and ARIMA model for water quality time series prediction.", Elsevier, vol 23, pp. 586-594

[92]. . Soman Saurabh S, Zareipour Hamidreza, Malik Om and Mandal Paras, 2010, "A Review of Wind Power and Wind Speed Forecasting Methods With Different Time Horizons".

[93]. Khalili Najmeh, Khodashenas Saeed Reza, Davary Kamran and Karimaldini Fatemeh, 2011, "Daily Rainfall Forecasting for Mashhad Synoptic Station using Artificial Neural Networks", International Conference on Environmental and Computer Science, vol.19, pp. 118-123.

[94]. Pan Tsung-Yi, Yang Yi-Ting, Kuo Hung-Chi, Tan YihChi, Lail Jihn-Sung, Chang Tsang-Jung, Lee Cheng-Shang, and Hsu Kathryn Hua, 2011, "Improvement of Statistical Typhoon Rainfall Forecasting with ANN-Based Southwest Monsoon Enhancement", Terr. Atmos. Ocean. Sci, Vol. 22, No. 6, pp. 633-645.

[95]. Joshi Jignesh, Patel Vinod M., 2011, "Rainfall-Runoff Modeling Using Artificial Neural Network (A Literature Review)", National Conference on Recent Trends in Engineering \& Technology.

[96]. El-Shafie Amr H., Shafie A. El-, Mazoghi Hasan G. El, Shehata A. and Taha Mohd. R., 2011, "Artificial neural network technique for rainfall forecasting applied to Alexandria, Egypt",

[97]. Mekanik F., Lee T.S. and Imteaz M. A., 2011, "Rainfall modeling using Artificial Neural Network for a mountainous region in West Iran".

[98]. Kaur Amanpreet, Singh Harpreet, 2011, "Artificial Neural Networks in Forecasting Minimum Temperature", International Journal of Electronics \& Communication Technology, Vol. 2, Issue 3, pp. 101-105.

[99]. El-Shafie A., Jaafer O. and Akrami Seyed Ahmad, 2011, "Adaptive neuro-fuzzy inference system based model for rainfall forecasting in Klang River, Malaysia",
International Journal of the Physical Sciences, Vol. 6(12), pp. $2875-2888$.

[100]. Tripathy Asis Kumar, Mohapatra Suvendu, Beura Shradhananda, Pradhan Gunanidhi, 2011. "Weather Forecasting using ANN and PSO", International Journal of Scientific \& Engineering Research, Volume 2, Issue 7, pp.1-5.

[101]. El-Shafie A, Noureldin A., Taha M. R., and Hussain A., 2011, "Dynamic versus static neural network model for rainfall forecasting at Klang River Basin, Malaysia", Hydrol. Earth Syst. Sci., vol. 8, pp. 6489-6532.

[102]. Geetha G.,. Selvaraj R Samuel, 2011, "Prediction of monthly rainfall in Chennai using back propagation neural network model", International Journal of Engineering Science and Technology, Vol. 3 No. 1, pp. 211-213.

[103]. Reshma T., Reddy K. Venkata, Pratap Deva, 2011, "Determination of Distributed Rainfall- Runoff Model Parameters Using Artificial Neural Network", International Journal of Earth Sciences and Engineering, Volume 04, No 06, pp. 222-224

[104]. Raju, M.Mohan,. Srivastava R. K, Bisht Dinesh C. S., Sharma H. C., and Kumar Anil, 2011, "Development of Artificial Neural-Network-BasedModels for the Simulation of Spring Discharge", Hindawi Publishing Corporation, Volume 2011, pp. 1-11.

[105]. Kavitha M.Mayilvaganan,.Naidu K.B, 2011, “ANN and Fuzzy Logic Models for the Prediction of groundwater level of a watershed", International Journal on Computer Science and Engineering, Vol. 3 No. 6, pp. 2523-2530.

[106]. El-shafie A., Mukhlisin M., Najah Ali A. and Taha M. $R$., 2011, "Performance of artificial neural network and regression techniques for rainfall-runoff prediction", International Journal of the Physical Sciences, Vol. 6(8), pp. 1997-2003.

[107]. Afshin Sarah, Fahmi Hedayat, Alizadeh Amin, Sedghi Hussein and Kaveh Fereidoon, 2011, "Long term rainfall forecasting by integrated artificial neural network-fuzzy logic-wavelet model in Karoon basin", Scientific Research and Essays, Vol. 6(6), pp. 1200-1208.

[108]. Siou1 Line Kong A, Johannet Anne, Borrell Valérie, Pistre Séverin, 2011, "Complexity selection of a neural network model for karst flood forecasting: The case of the Lez Basin (southern France)", Journal of Hydrology, vol. 403, pp. 367-380.

[109]. Saima H., Jaafar J., Belhaouari S., Jillani T.A., 2011 "Intelligent Methods for Weather Forecasting: A Review", IEEE,

[110]. Sawaitul Sanjay D., Prof. Wagh K. P., Dr. Chatur P. N., 2012, "Classification and Prediction of Future Weather by using Back Propagation Algorithm-An Approach", International Journal of Emerging Technology and Advanced Engineering, Volume 2, Issue 1, pp. 110-113.

[111]. Karmakar, S., Kowar, M. K., Guhathakurta, P., Evolving and Evaluation of 3LP FFBP Deterministic ANN Model for District Level Long Range Monsoon Rainfall Prediction, J. Environmental Science \& Engineering, , National Environmental Engineering Research Institute, Nagpur, INDIA, ISSN 0367-827, Vol 51, No. 2, pp. 137-144, April 2009. URL: http://www.neeri.res.in/jese.html. 
[112] Karmakar, S., Kowar, M. K., Guhathakurta, P., Development of an 8-Parameter Probabilistic Artificial Neural Network Model for Long-Range Monsoon Rainfall Pattern Recognition over the Smaller Scale Geographical Region -District, IEEE Computer Society, IEEE Xplore 2.0, DC, USA, ISBN 978-0-7695-3267-7, pp. 569-574, July 2008. http://www2.computer.org/portal/web/csdl/doi/10.1109/ICE TET.2008.225.

http://portal.acm.org/citation.cfm?id=1445475

[113] Karmakar, S., Kowar, M. K., Guhathakurta, P., Development of Artificial Neural Network Models for Long-Range Meteorological Parameters Pattern Recognition over the Smaller Scale Geographical Region-
District, IEEE Xplore 2.0, IEEE Computer Society, Washington, DC, USA, ISBN 978-1-4244-2806-9, pp. 1-6, Dec. 2008 .

URL:http://ieeexplore.ieee.org/xpl/freeabs_all.jsp?isnumber $=4798312 \&$ arnumber $=4798370 \&$ count $=184$ \&index $=54$.

[114]. Karmakar, S., Kowar, M. K., Guhathakurta, P., LongRange Monsoon Rainfall Pattern Recognition \& Prediction for the Subdivision 'EPMB' Chhattisgarh Using Deterministic \& Probabilistic Neural Network, IEEE Xplore 2.0, IEEE Computer Society, Washington, DC, USA, ISBN 978-0-7695-3520-3, pp. 376-370, Feb. 2009. http://www2.computer.org/portal/web/csdl/doi/10.1109/IC APR.2009.24.

Table 1. Performance of BPN in deterministic forecast during training and independent Period

\begin{tabular}{|c|c|c|c|c|c|c|c|c|c|c|c|c|}
\hline \multirow{2}{*}{ Data } & \multicolumn{5}{|c|}{ Training period (1951-1991) } & \multicolumn{3}{c|}{ Independent/testing period (1991-2004) } \\
\cline { 2 - 12 } & Mean & SD & MAD & $\begin{array}{c}\text { SD } \\
\text { \% of } \\
\text { mean })\end{array}$ & $\begin{array}{c}\text { MAD } \\
(\% \text { of } \\
\text { mean })\end{array}$ & CC & Mean & SD & MAD & $\begin{array}{c}\text { SD } \\
(\% \text { of } \\
\text { mean })\end{array}$ & $\begin{array}{c}\text { MAD } \\
\text { (\% of } \\
\text { mean })\end{array}$ & CC \\
\hline $\begin{array}{c}\text { Normalized } \\
\text { De- } \\
\begin{array}{c}\text { Normalized } \\
\text { (in mm) }\end{array}\end{array}$ & 0.62 & 0.03 & 0.02 & 5.51 & 3.21 & 0.6 & 0.62 & 0.03 & 0.02 & 5.50 & 2.96 & 0.8 \\
\hline
\end{tabular}

